\title{
EXPERIENCIAS DE TRATAMIENTO DE MÁRGENES DE CARRETERAS EN LA COMUNIDAD DE MADRID
}

\author{
(EXPERIENCES ON ROAD EDGE TREATMENT IN THE COMMUNITY \\ OF MADRID)
}

Modesto Franch Mariner, Ingeniero de Montes

Fecha de recepción: 27 - IV -93

Dirección General de Carreteras - Comunidad de Madrid

ESPAÑA

\section{RESUMEN}

Se describen en estas páginas las pautas y criterios generales seguidos en los Proyectos y Obras de Tratamiento de Márgenes de Carreteras en la Comunidad de Madrid, que tienen por objeto la integración de la carretera en el entorno circundante. Indica las actuaciones en márgenes laterales, medianas e intersecciones, asi como las medidas correctoras para reducir los impactos, nuevas técnicas de estabilización de taludes, y labores de conservación $y$ mantenimiento.

\section{SUMMARY}

This article describes the guidelines and general criteria followed in the projects and works of road edge treatment in the Community of Madrid which are aimed at integrating the road in the surroundings. The works on lateral edges, median strips and junctions as well as impact reducing corrective measures, new techniques in bank stabilization and conservation and maintenance works are presented

\section{INTRODUCCIÓN}

Actualmente la construcción de una carretera, debido a las mayores exigencias de trazado en cuanto a radio mínimo de las curvas, pendientes, anchuras y taludes, hacen difícil la adaptación al terreno, llevando consigo grandes movimientos de tierra, que modifican el entorno por el que discurre. Esto genera un fuerte impacto en el medio ambiente, que es necesario corregir mediante obras de restauración y acondicionamiento, con planteamientos funcionales y estéticos, que integren la carretera en el paisaje y mejoren el funcionamiento de la vía.

\section{IDENTIFICACIÓN Y CARACTERIZACIÓN DE LOS IMPACTOS}

Para determinar las principales acciones sobre el suelo conviene diferenciar dos fases:

- Fase de construcción.

- Fase de funcionamiento.

\section{Acciones}

- Fase de construcción:

- Alteraciones debidas a la utilización de maquinaria. 


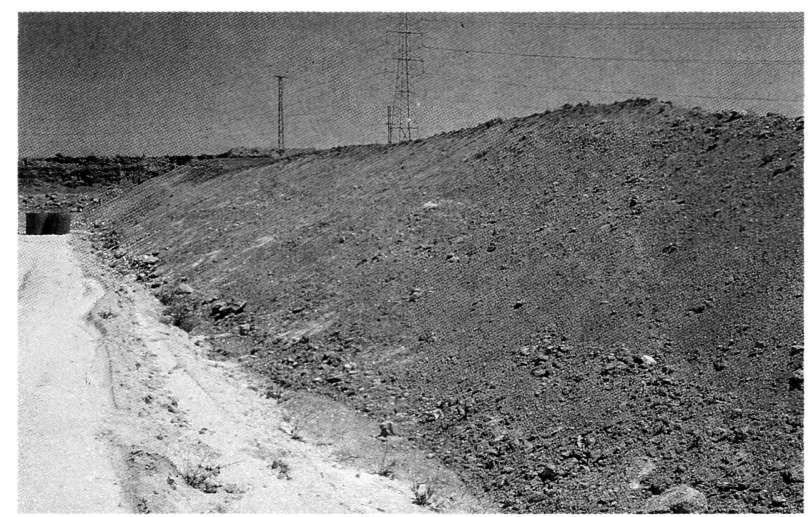

Foto 1. Talud sin tratar en tierra

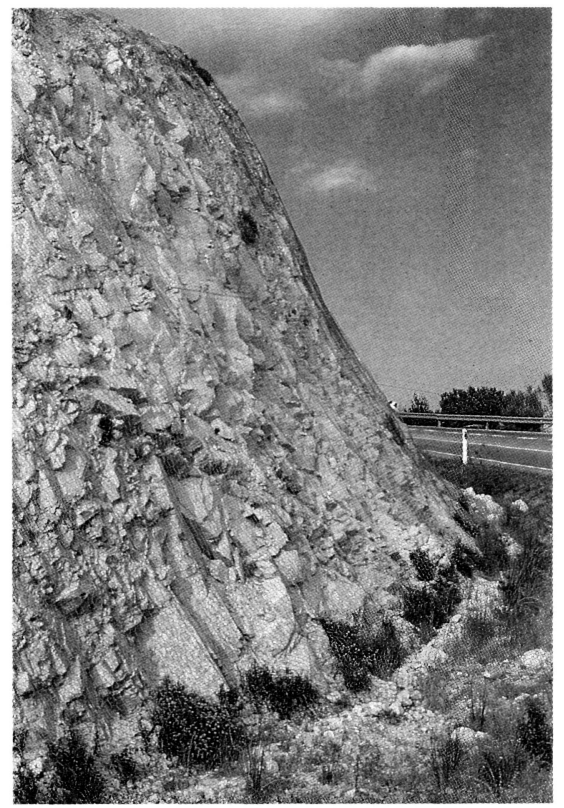

Foto 2. Talud sin tratar en roca.

- Movimiento de tierras:

- Escarificado de la capa vegetal. Pérdida de suelo.

- Excavaciones a cielo abierto.

- Desmontes.

- Voladuras.

- Apertura de cimentaciones.

- Terraplenes y rellenos.

- Acopios.

- Vertidos.

- Recubrimiento e impermeabilización de grandes superficies.

Fase de funcionamiento:

(c) Consejo Superior de Investigaciones Científicas Licencia Creative Commons 3.0 España (by-nc)
- Alteraciones debidas al propio mantenimiento y a la corrección de posibles deficiencias del Proyecto.

- Movimiento de tierras:

- Limpieza de cunetas.

- Rectificado de taludes por efecto de los deslizamientos.

- Rectificado de terraplenes y obras de fábrica.

\section{Efectos}

\section{Fase de construcción}

Se producen cambios en los procesos erosivos al variar las pendientes y crear superficies desnudas desprovistas de vegetación, como los taludes, en los que se pueden dar procesos de inestabilidad.

(Fotos 1 y 2).

De no tomar medidas, se producirá una disminución de la productividad al eliminar el horizonte húmico, seguido de la eliminación de especies vegetales y fauna.

Aparte de estos efectos, hay que añadir los de actividades anexas a la vía, como las canteras de extracción de áridos, y la producción de materiales residuales que hay que trasladar a vertedero.

\section{Fase de funcionamiento}

Aparición de erosión en taludes desnudos, debido a la ausencia de vegetación protectora.

Las gotas de lluvia producen procesos de escorrentía superficial, con el consiguiente arrastre de materiales y pérdida de elementos finos y nutrientes, que dificulta la regeneración vegetal protectora.

Los efectos inducidos en una y otra fase los podemos resumir en:

- Erosión.

- Deslizamientos superficiales.

- Desprendimientos y caídas de bloques desde taludes.

- Pérdida de suelo agrícola/productividad.

- Disminución de la capacidad de cobertura en taludes.

\section{TRATAMIENTOS}

Tienen por objeto la integración de la carretera en su paisaje circundante, mediante planteamientos funcio- 




Foto 3. Glorieta tratada

nales y estéticos, que tendrán como fin último mejorar el funcionamiento de la vía.

Márgenes laterales: A lo largo de la traza cambia la topografía, y en función de ésta sus tratamientos.

Se tendrá en cuenta una zona de seguridad, que será como mínimo la altura máxima prevista para los árboles, y una zona de desprendimiento cuando la vía transcurra en trinchera. No se plantarán en estas zonas especies arbóreas.

Las curvas se "dibujarán" mediante alineaciones de árboles, y/o arbustos en su parte exterior.

Los cambios de rasante en curva se indicarán mediante alineaciones de árboles, que aumentarán en altura de arriba a abajo.

Con el fin de evitar el golpe de viento al salir de una trinchera, colocaremos a ambos lados pantallas de árboles de hoja perenne, ramificados desde la base y con densidad de plantación decreciente.

En los desmontes y terraplenes es donde el tratamiento presenta mayores problemas.

Nuestro objetivo consiste en implantar una cubierta vegetal en un talud artificial, ya sea directamente o adoptando medidas complementarias de apoyo.

Las superficies a tratar presentan pendientes superiores a las que el terreno tenía antes de realizar el movimiento de tierras necesario para la explanación. Esta circunstancia afecta tanto a la insolación recibida por el talud, como al límite de estabilidad de los materiales que lo constituyen.

La fuerte inclinación de los taludes, agudiza la diferente exposición de la luz solar, dándose exposiciones opuestas -solana umbría- en un mismo talud.

Los afloramientos rocosos son otra causa limitativa para el arraigo de la vegetación.

Finalmente, las condiciones edáficas del suelo son factores determinantes para el establecimiento de las plantas. La remodelación del terreno empleando maquinaria pesada, es causa de su intensa compactación. Carecen de horizonte húmico, y el pH presenta valores más altos, o más bajos, que en el resto de la zona. En una primera fase de estabilización, la técnica más económica y eficaz es la hidrosiembra, apoyada con medidas complementarias cuando las condiciones lo requieran. En una segunda fase, como complemento del tapiz herbáceo se realizarán plantaciones de arbustos.

Medianas: Tres funciones principales destacamos en el tratamiento de las medianas:

- Eliminar la visión del tráfico por el carril contrario.

- Proteger del deslumbramiento.

- Indicar mejor la forma de la traza marcando de lejos las curvas, cambios de rasante e intersecciones. 
Se respetará una franja de 1,5 a $2 \mathrm{~m}$ del borde de la calzada, como medida de seguridad, y se evitará la monotonía en la distribución de las especies, teniendo en cuenta que la percepción es dinámica y no estática.

Intersecciones: La principal misión de las plantaciones es servir como punto de referencia, que marque la intersección a cierta distancia.

Aquí cobra capital importancia el no interferir la visibilidad.

En las glorietas suburbanas tipo "Roundabout", la función del tratamiento paisajístico es contribuir a un mejor y más seguro funcionamiento de los movimientos previstos (foto 3 ).

Por el contraste entre su forma circular, y la linealidad de la vía, se convierten en focos visuales de las carreteras confluyentes, lo que hace que sean un lugar ideal para la realización de tratamientos paisajísticos, que tiendan a reforzar su carácter de hito dentro de la vía.

Estos tratamientos deberán contribuir a:

- Favorecer la percepción lejana de la glorieta.

- Identificar el lugar como punto de ruptura del itinerario.

- Favorecer la orientación del usuario del anillo.

- Mejorar el confort físico y psíquico del conductor.

Por otro lado tendremos en cuenta las siguientes restricciones:

-Alrededor de la glorieta, y en el islote central, no se dispondrán masas de vegetación compacta que impidan la visión de seguridad.

-Las isletas deflectoras estarán exentas de masas de vegetación, que dificulten la visibilidad sobre el anillo, entradas, salidas y señalización.

\section{MEDIDAS CORRECTORAS PARA REDUCIR LOS IMPACTOS}

Vegetación: Más que medidas correctoras para reducir los impactos, habrá que aplicar medidas preventivas, reduciendo al máximo la superficie alterada, recuperando ésta, creando las condiciones óptimas de forma que posibilite a corto plazo la implantación de especies herbáceas que creen el sustrato necesario para que, a medio y largo plazo, sea posible la instalación de la vegetación autóctona inicial.

En los casos en que este proceso natural no sea lo suficientemente rápido para evitar la erosión y como consecuencia el aumento de la degradación del suelo, habrá que ayudar a los procesos naturales con plantaciones y siembras.

Suelos: Las alteraciones más importantes sobre los suelos las podemos reducir a tres:

- Aumento de la erosión.

- Pérdida del horizonte húmico.

- Compactación en los alrededores de la vía.

Las medidas correctoras empezarán con la recogida y acopio en buenas condiciones del suelo que tenga valor agrológico, para su posterior utilización en labores de revegetación.

Cuando esto no sea posible, se realizarán las enmiendas pertinentes para dar la fertilidad adecuada y así hacer posible la implantación de una cubierta vegetal, que sirva como primer paso para la creación del horizonte húmico necesario, y que en fases sucesivas se pueda implantar la flora autóctona.

Estabilización de taludes: La superficie de desmontes y terraplenes desprovista de suelo está sometida a procesos de erosión y meteorización, que favorecen la aparición de regueras y cárcavas, ocasionando la ruina del talud. La implantación de una cubierta vegetal es el método menos caro para conseguir su estabilización, ya que ésta favorece la formación de un horizonte con humus sobre la superficie inerte, creando las condiciones para el desarrollo de las especies autóctonas en un espacio corto de tiempo.

La naturaleza de los taludes es hostil al arraigo de las plantas, lo que exige la aplicación de medidas correctoras para:

- Evitar el arrastre de las semillas por la erosión hídrica y eólica.

- Permitir que el agua penetre en el suelo durante el período de implantación.

- Disminuir la evapotranspiración.

- Proporcionar un sustrato favorable para la germinación de las semillas. 
Fotos 4, a y b. Proceso de hidrosembrado.

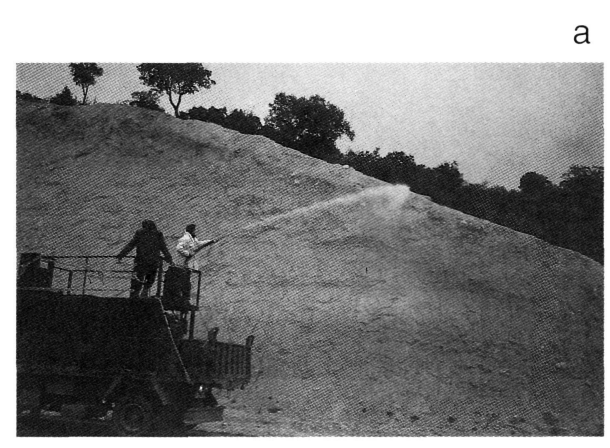

b

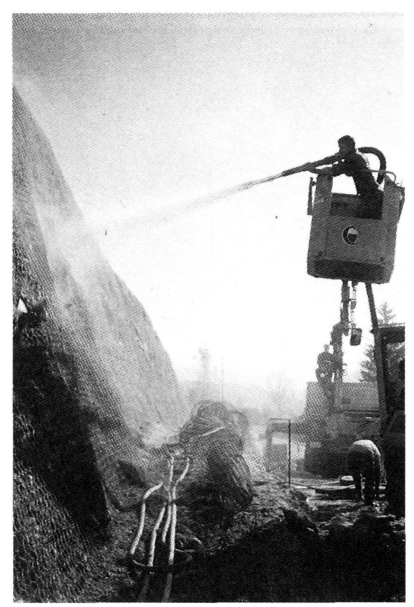

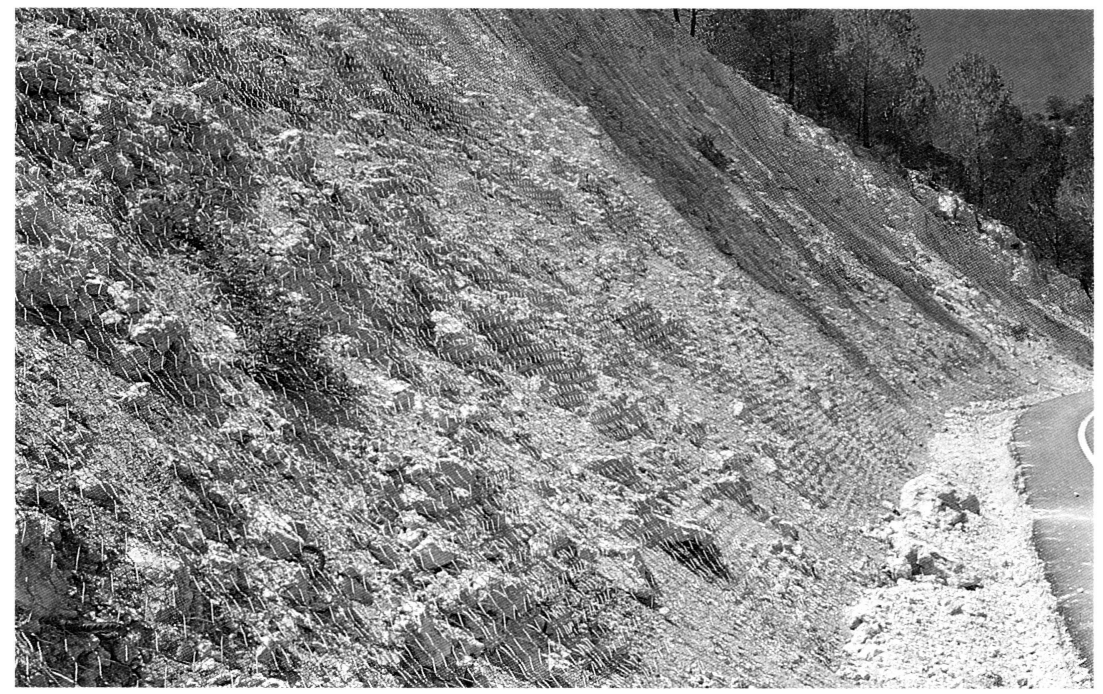



Estas condiciones las podemos lograr con la hidrosiembra, acompañada de medidas complementarias cuando las condiciones del medio lo requieran. Para ello usaremos una mezcla de productos que podemos resumir (foto 4) en:

- Estabilizadores: Su papel es fijar los componentes de la hidrosiembra con los agregados finos de suelo por aglomeración física de las partículas.

- Mulch: Se define como toda cubierta superficial del suelo, ya sea orgánica o inorgánica, que tenga un efecto protector. Protege las semillas, reduce la evapotranspiración, y absorbe el impacto directo de las gotas de lluvia.

- Fertilizantes: Compuestos por abono tipo NPK de

(c) Consejo Superior de Investigaciones Científicas liberación lenta, y ácidos húmicos y fúlvicos.

- Semillas: Compuestas por una mezcla de gramíneas y leguminosas.

- Agua.

Esta mezcla se proyecta sobre el talud mediante una máquina hidrosembradora.

La operación se realiza en dos fases: fase de siembra, en la que se proyecta la mezcla completa; fase de tapado, proyectando mulch y estabilizador.

En taludes muy inestables, previo a la łidrosiembra, se colocará anclada en coronación y adosada al talud mediante grapas una malla metálica. (Foto 5). 


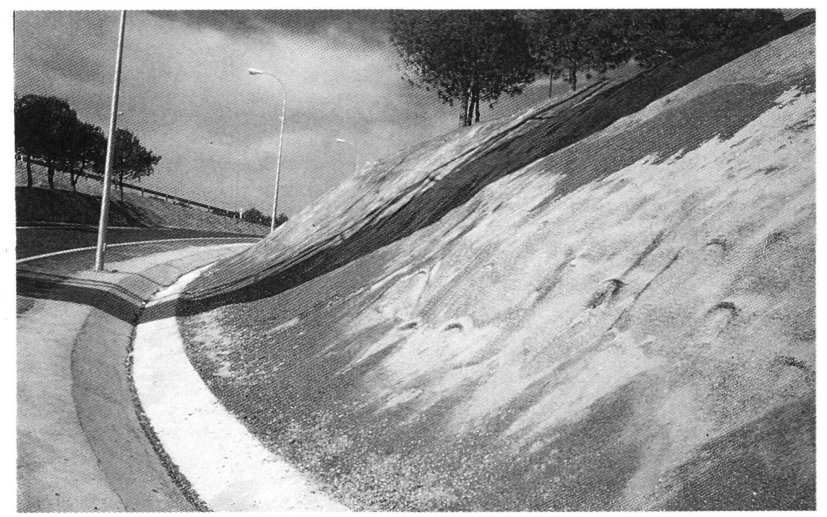

Foto 6. Estera de poliamida. (Talud pronunciado sin desprendimiento).

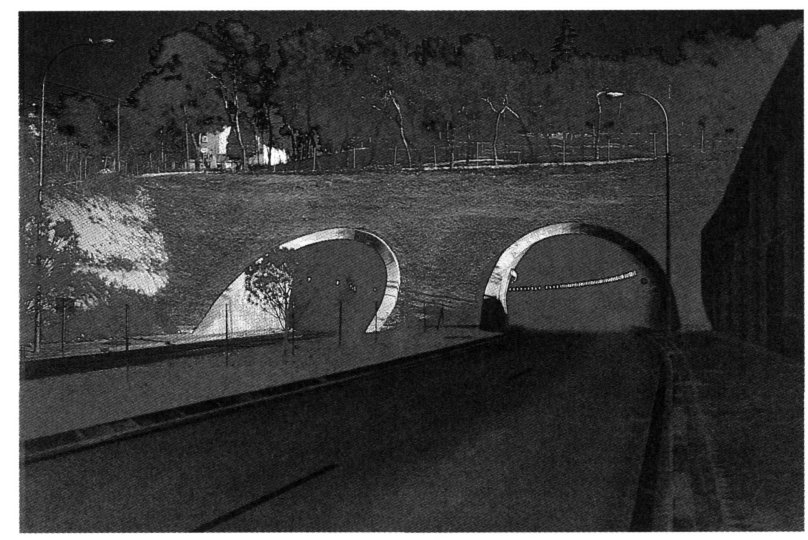

Foto 8. Estera de poliamida colonizada por la hierba.

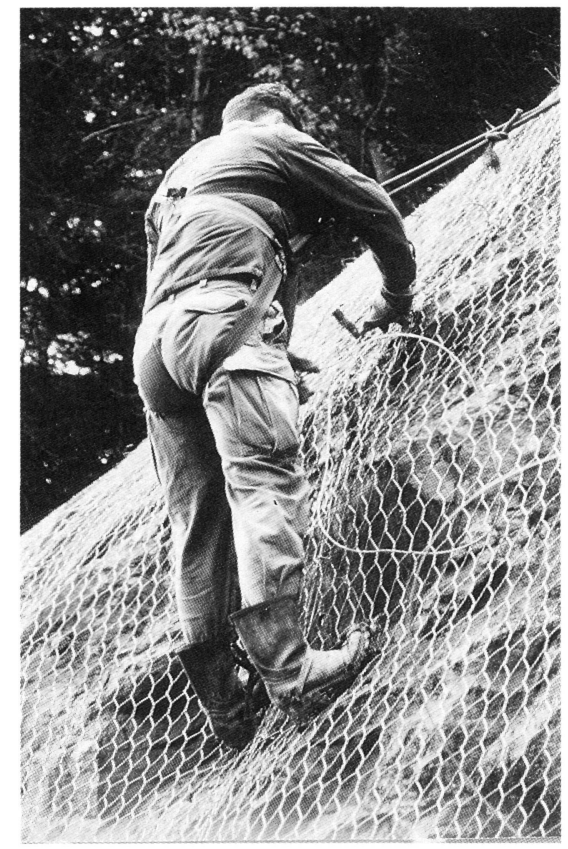

Foto 10. Colocación de mallazo. (Talud con desprendimientos).

(c) Consejo Superior de Investigaciones Científicas Licencia Creative Commons 3.0 España (by-nc)

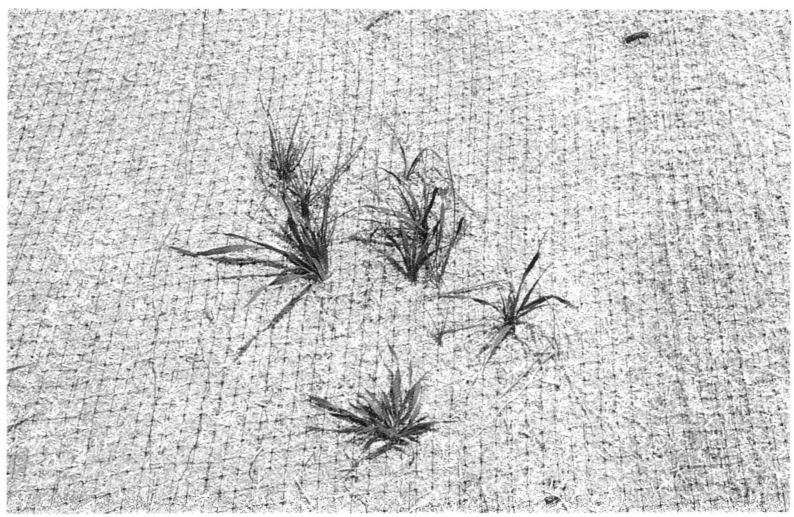

Foto 7. Estera orgánica. (Talud pronunciado sin desprendimiento).

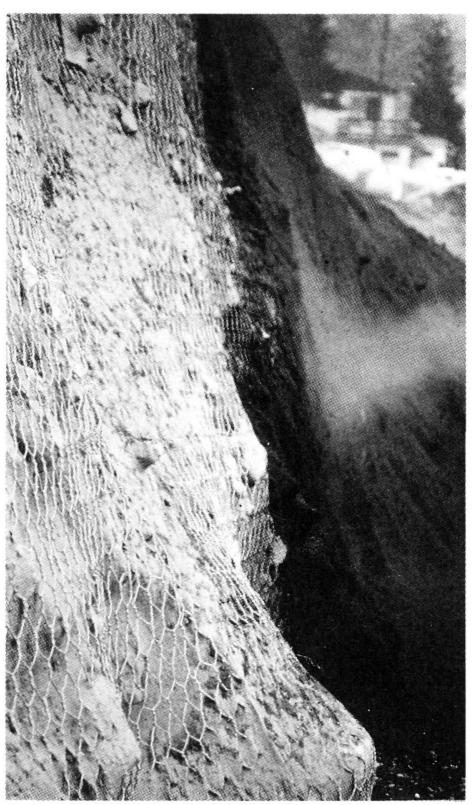

Cuando la pendiente sea muy pronunciada facilitará, en gran medida, el asentamiento de la vegetación, así como colocar una estera tridimensional de poliamida con el 95\% de espacio hueco donde se alojará la mezcla, evitando así su desprendimiento. (Fotos 6, 7 y 8$)$.

En las carreteras de la sierra, los riesgos de desprendimiento no pueden evitarse sólo con un diseño adecuado de los taludes, tendiendo más la pendiente, construyendo bermas, etc, ya que a causa de la nieve tienen lugar procesos de reptación y solifluxión.

El primero consiste en un movimiento lento del suelo o de la regolita sobre los taludes pendiente abajo a causa de la gravedad, acentuado=por una expansión y contracción, alterando el equilibrio mecánico interno del material debido al hielo-deshielo y a la alternancia de humedad-sequía. 
Perfil transversal. Tratamiento para desmonte o terraplén.

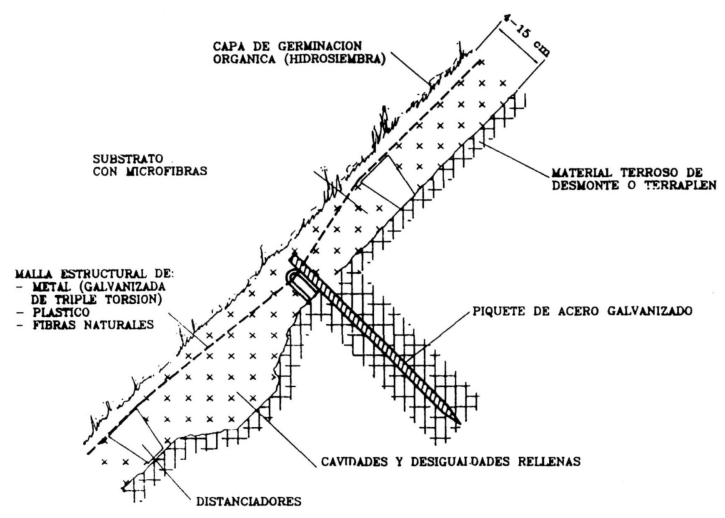

\section{Perfil transversal. Tratamiento para desmonte rocoso.}

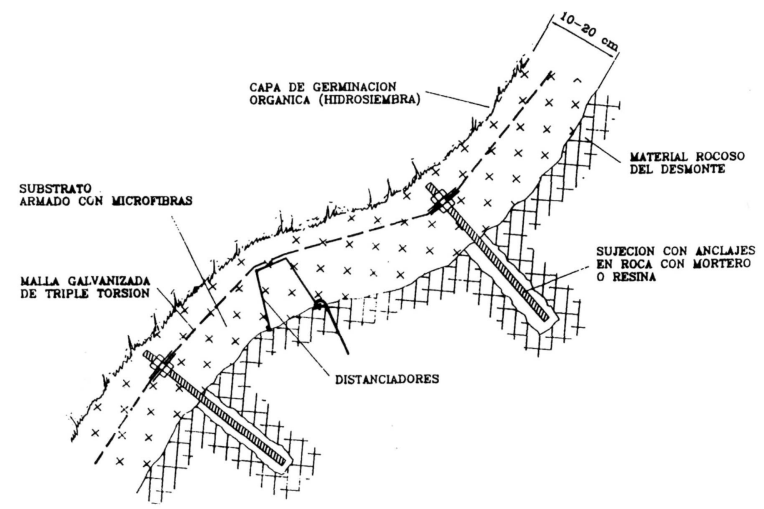

Instalación malla galvanizada

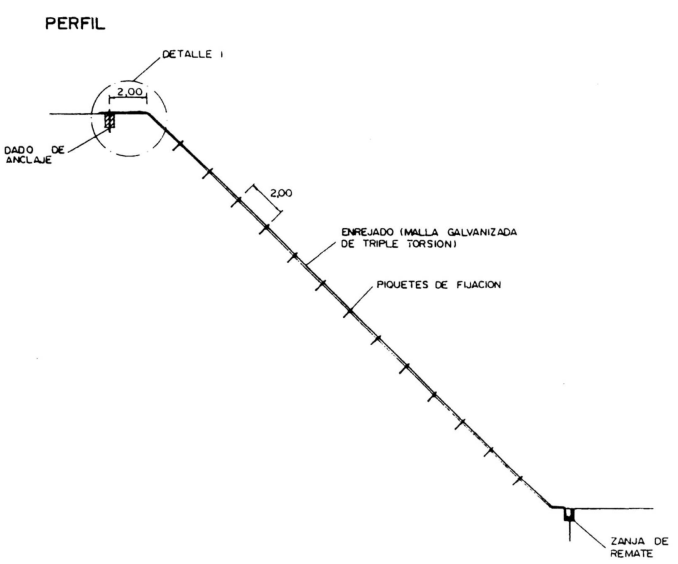

Fig. A. Detalle de sujeción del mallazo

Detalle de alzado



Detalle 1. Remate superior

(c) Consejo Superior de Investigaciones Científicas 


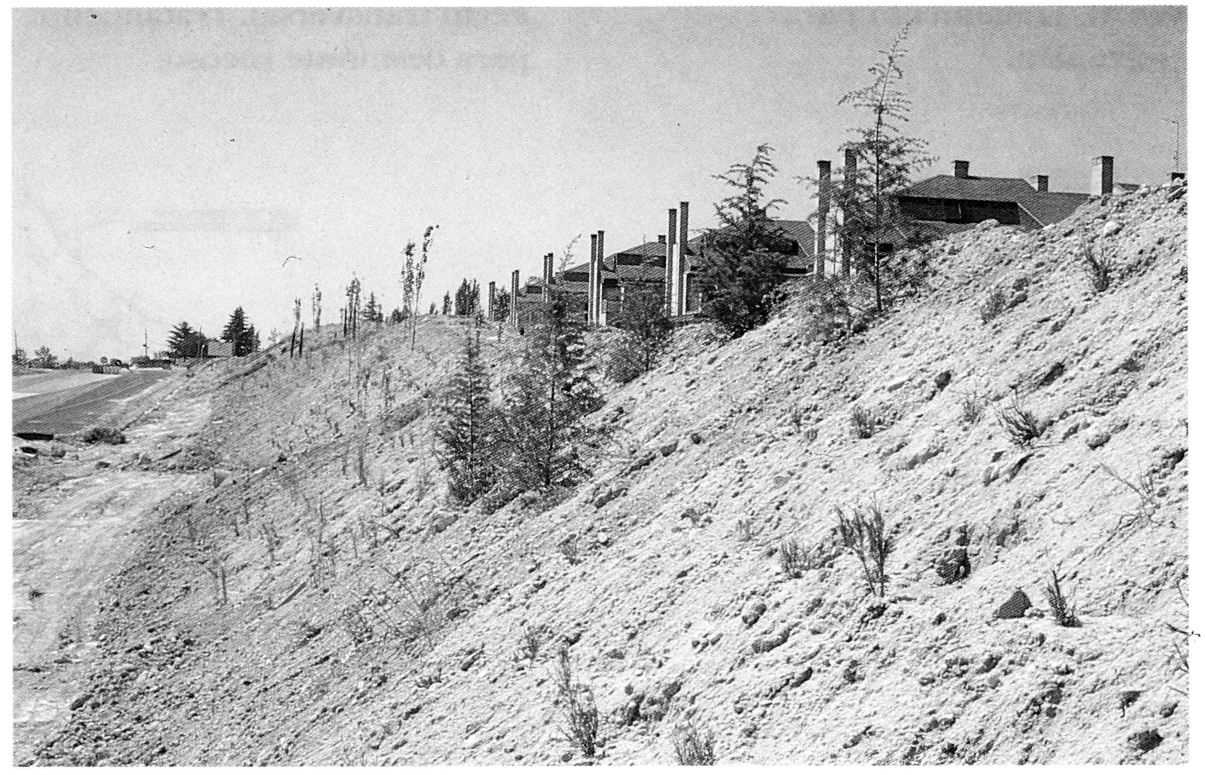

Foto 11. Caballones para protección acústica.

El segundo es un mecanismo gravitatorio propio de climas fríos. Es un movimiento complejo, causado por el hielo-deshielo, de forma que se expande el material disminuyendo su cohesión. El deshielo proporciona el agua necesaria para que el material llegue a alcanzar el límite líquido. Este proceso alcanza a un manto poco profundo pero extenso.

Como medidas correctoras, construiremos una cuneta de coronación para desviar el agua de escorrentía, pondremos los drenes que sean necesarios y seguidamente colocaremos una malla metálica de triple torsión galvanizada, anclada en la coronación y en la base, para impedir el desprendimiento de los materiales. A partir de aquí ya se puede intentar implantar la vegetación empezando con la hidrosiembra con varias fases de tapado, logrando así una capa espesa, donde pueda iniciarse la germinación de las semillas. (Fotos 9, 10 y Fig. A).

Para acelerar el proceso de implantación de la cubierta vegetal en estos taludes se ha ensayado, con resultados satisfactorios, una técnica similar al gunitado. Consiste en proyectar sobre el talud, al que previamente hemos colocado la malla, una mezcla de turba, tierra vegetal, paja, acondicionador de suelos, fertilizante, cemento portland, un corrector de $\mathrm{pH}$, semillas de gramíneas y leguminosas, con un espesor de $10 \mathrm{~cm}$. La aplicación se hace por vía seca, reuniéndose el agua de fraguado en la boquilla de salida al exterior, por intermedio de un anillo perforado.

(c) Consejo Superior de Investigaciones Científicas
Antes de transcurrir un mes de la aplicación, ya se ha observado la "nascencia" de las primeras plántulas homogéneamente por toda la superficie.

Protección acústica: Las pantallas sónicas tienen como finalidad amortiguar los niveles sonoros producidos por el funcionamiento de la vía.

Su diseño puede ser muy variado: desde prefabricadas a simples caballones de tierra. La vegetación produce una disminución sonora poco acusada. Si se dispone de espacio suficiente, una buena solución será la construcción de un dique de tierra de perfil trapezoidal, tratado con plantaciones de especies perennes y ramificadas desde la base, colocadas con la mayor densidad posible.

Paisaje: Todas las medidas anteriores, bien aplicadas, contribuirán a la integración paisajística de la vía, aumentando la seguridad, ayudando al seguimiento óptico de la carretera, evitando deslumbramientos, disminuyendo el riesgo de desprendimiento de materiales a la calzada, etc.

En cuanto al diseño, la singularidad de cada vía, tanto en lo que corresponde al trazado como al entorno afectado, hace que cada caso tenga una solución distinta. En general, en zonas suburbanas, más humanizadas, se tenderá a diseños más cuidados, ineluso ajardinados. Por el contrario, en zonas claramenterurales, el diseño debe adaptarse a la textura y color del paisaje, dándole un tratamiento acorde con las zonas circundantes. 


\section{CONSERVACIÓN Y MANTENIMIENTO}

De poco nos serviría el Tratamiento de Márgenes, si no va acompañado de un adecuado mantenimiento. Éste se realizará desde el doble aspecto funcional y estético, y tendrá como fin contribuir al buen funcionamiento de la vía.

\section{Funcional:}

- Mantener limpias las cunetas evitando que la vegetación obstaculice la libre circulación del agua.

- Controlar la invasión por la vegetación de zonas mineralizadas.

- Mantener la visibilidad y evitar la obstaculización de las señales de tráfico.

- Mantener la vegetación en los taludes para evitar que sean erosionados.

- Limitar el riesgo de incendio y su propagación.

\section{Estéticos:}

- Lograr que los márgenes estén limpios, no dando sensación de abandono.

- Cuidar las áreas frecuentadas por peatones en los aledaños de la carretera.

- Controlar el crecimiento de la vegetación.

- Realzar el trazado de la vía y sus elementos más característicos.

Las labores fundamentales que se llevarán a cabo son las siguientes:

- Riegos.

- Siegas.

- Podas.

- Escardas y binas.

- Mantenimiento de alcorques.

- Instalación de vientos y tutores.

- Abonados.

- Tratamientos fitosanitarios.
La siega es en la mayoría de los casos la labor más importante, y la menos aleatoria respecto a los resultados obtenidos.

Arcén: La frecuencia de segado variará entre una y cinco veces al año, dependiendo de las condiciones meteorológicas. El objetivo es que la hierba no sobrepase los $20 \mathrm{~cm}$ de altura.

Medianas: El mismo tratamiento que en el arcén.

Cunetas: Debe realizarse, tantas veces como sea necesario, para evitar que se obstaculice la libre circulación del agua. El desbroce químico debe ser realizado con gran prudencia, teniendo en cuenta los riesgos de contaminación que se pueden producir.

Taludes: En desmontes con pendiente suave es factible la utilización de maquinaria. El objetivo es que la hierba no sobrepase los $40 \mathrm{~cm}$. En los de altura elevada, y con fuerte pendiente, se segará hasta los $3 \mathrm{~m}$ de altura.

En terraplenes se tendrá en cuenta su visibilidad desde la carretera; en caso de que sean particularmente visibles se segará tantas veces como la hierba alcance 40 centímetros.

En cuanto al riego, es preciso proporcionar agua a las plantas al menos durante los tres primeros años que siguen a la plantación. Para que sea efectivo, se mantendrán en todo momento los alcorques en perfecto estado. La frecuencia será de seis a ocho riegos al año como mínimo. Dependiendo del tipo de planta las dosis serán las siguientes:

- Árboles: de 25 a 30 1/unidad.

- Arbustos: de 10 a 20 1/unidad.

- Subarbustos y matas: de 5 a 10 1/unidad.

El resto de las labores, aunque pueden llegar a ser fundamentales, no exigen un calendario tan estricto. 